\title{
Retraction Note: Functional screen analysis reveals miR-3142 as central regulator in chemoresistance and proliferation through activation of the PTEN- AKT pathway in CML
}

Lifen Zhao, Yujia Shan, Bing Liu, Yang Li and Li Jia

Retraction to: Cell Death and Disease https://doi.org/10.1038/cddis.2017.223

published online 25 May 2017

The Editors-in-Chief have retracted this article because the PTEN-shRNA panel in Fig. $6 \mathrm{~d}$ appears to be the same as the Anti3142+PTEN-shRNA panel in Fig. 6h. The Editors-in-Chief therefore no longer have confidence in the integrity of the data in Fig. 6. All of the authors disagree with this retraction.

Published online: 12 February 2020 